\title{
LA GERENCIA DEL CONOCIMIENTO COMO FUENTE DE VENTAJA PARA DESARROLLAR LAS MYPES
}

\author{
Pedro Tito Huamani* \\ E-mail: pedrotito18@hotmail.com
}

\begin{abstract}
RESUMEN
Los resultados de la investigación demuestran que la Gerencia del Conocimiento es un modelo de gestión innovador y creativo para las organizaciones empresariales, sean éstas micro, pequeñas, medianas y/o grandes empresas. A diferencia de los últimos, en los primeros (micro y pequeñas empresas o MYPEs), dadas las limitaciones financieras y de gestión para la implementación de este modelo, debe seguirse la tendencia a la personificación; vale decir, una gestión caracterizada por el contacto permanente con sus colaboradores, en la que la tecnología ayude a comunicar sus conocimientos y no solo a almacenarlos.
\end{abstract}

A nivel interno todavía no están dadas las condiciones -clima laboral en los talleres y formación académica de los propietarios- para gerenciar las MYPEs sobre la base de los conocimientos acumulados a través de sus colaboradores. Esto se refleja en el celo que todavía subsiste entre los trabajadores, dificultando compartir conocimientos, habilidades y destrezas aunque están convencidos que para desarrollar la organización necesariamente deben interactuar e intercambiar conocimientos.

De la misma forma, a partir de los resultados de la investigación, se concluye que la adquisición de nuevos conocimientos para desarrollar su trabajo tiene una alta valoración entre los trabajadores y propietarios de las MYPEs. Creemos que este aspecto es importante para internalizar los valores de interacción humana y trabajo en equipo.

Palabras clave: Dato, información, conocimiento, innovación, MYPE.

\section{ABSTRACT}

The results of the investigation demonstrate that the Management of the Knowledge is an innovative and creative administration model for the managerial organizations: micro, small, medium and big companies. Contrary to the last ones, in the first ones (micro and small companies or Mypes), given the financial and administrative limitations for this models' implementation, the trend should be continued to the personification; it is worth to say, an administration characterized by the permanent contact with their collaborators, in which the technology would help to communicate its knowledge and not only to store them.

At internal level, the conditions are not still given - labor climate in the shops and the proprietors' academic formation - to manage the Mypes on the base of the knowledge accumulated through their collaborators. This is reflected in the zeal that subsists still among the workers, hindering to share

* Magíster y Licenciado en Administración. Docente Permanente de la UNMSM, Docente de Post Grado en UNMSM, UNA, UNSCH, UNLG y UNP. Consultor en Gerencia del Potencial Humano y Comportamiento Organizacional. 
knowledge, abilities and dexterities; although they are convinced they have to interact and exchange information to develop the organization.

In the same way, starting from the results of the investigation, it is concluded that the acquisition of new knowledge to develop its work has a high value between the workers and proprietors of the MYPEs. We believe this aspect is important to internalize the human interaction values and the team work.

Keywords: I date, information, knowledge, innovation, MYPE.

\section{INTRODUCCIÓN}

Un nuevo paradigma viene sacudiendo a las organizaciones empresariales en el mundo. $Y$ es que a diferencia de décadas pasadas, donde la acumulación en mayor infraestructura (instalaciones, equipos), así como en mayores inversiones en activos tangibles, fueron sinónimos de fortaleza y solidez económica empresarial. Hoy en día, las organizaciones exitosas son los que reflejan activos tangibles significativamente reducidos pero que -en contraposición- se preocupan en potenciar sus activos intangibles. Ejemplo: la Corporación Microsoft de Bill Gates. En ese contexto, son los conocimientos que las organizaciones adquieren a través de su gente los que se constituyen en ventajas diferenciales y que vienen siendo aprovechados para garantizar su éxito futuro.

Ahora bien, el conocimiento es inherente a la persona humana. Las personas siempre fueron el componente básico y primordial de las organizaciones, sean estas micro, pequeñas, medianas o grandes empresas. Tal y como apuntan algunos especialistas (Drucker, 1993), las organizaciones del futuro sólo podrán adquirir y mantener ventajas competitivas mediante el uso adecuado de la información y, sobre todo, del conocimiento. El tipo de conocimiento que puede aportar ventajas competitivas a una organización abarca un rango muy amplio que incluye desde aquel que se puede patentar hasta el conocimiento sobre las necesidades de los clientes, pasando por el conocimiento que permite mejorar el servicio de atención post venta u optimizar los procesos de producción.

Así pues, si una organización desea ser competitiva de forma sostenida en el tiempo deberá identificar, crear, almacenar, transmitir y utilizar de forma eficiente el conocimiento individual y colectivo de sus trabajadores con el fin de resolver problemas, mejorar procesos o servicios y, sobre todo, aprovechar nuevas oportunidades de negocio.

\section{MARCO TEÓRICO}

¿Por qué las organizaciones empresariales, incluidas las MYPEs, tendrían que avanzar en sus modelos de gestión, a tal grado de validar los conocimientos de sus colaboradores, aunque éstos no se reflejen en activos tangibles al final de un periodo económico?

Precisamente son estos recursos intangibles los que explican el valor de mercado de la empresa, y en ellos se consideran no sólo los recursos tangibles de la empresa, sino también los recursos intangibles -recursos que, la mayor parte de las veces, no quedan registrados en los libros contables de la empresa-, principalmente el capital intelectual. Prescindiendo del componente especulativo, existe consenso en considerar la importancia de dichos componentes intangibles, los cuales permitirían la obtención de ventajas competitivas sostenibles en el tiempo. En este sentido, intangibles tales como la cualificación de los colaboradores de una organización estarán directamente relacionados con el valor de mercado de la misma.

\section{DIFERENCIA ENTRE DATO, INFORMACIÓN Y CONOCIMIENTO}

Es importante, para los propósitos de esta investigación tener claridad acerca de estos tres conceptos; dado que en nuestro medio se suelen utilizar indistintamente y esto puede llevar a una interpretación errónea de la Gestión del Conocimiento. Una primera aproximación a la diferenciación de tales conceptos es que los datos están localizados en el mundo, en tanto que el conocimiento está localizado en cualquier agente, sean personas u organizaciones. La información adopta un papel mediador entre ambos conceptos.

\section{DATO}

Veamos algunos conceptos relacionados:

"Un dato es un conjunto discreto de factores objetivos sobre un hecho real. Dentro de un 
contexto empresarial, el concepto de dato es definido como un registro de transacciones. Un dato no dice nada sobre el por qué de las cosas, y por sí mismo tiene poca o ninguna relevancia o propósito. Los datos describen únicamente una parte de lo que pasa en la realidad y no proporcionan juicios de valor o interpretaciones, y por lo tanto no son orientativos para la acción. La toma de decisiones se basará en datos, pero estos nunca dirán lo que se ha de hacer. A pesar de todo, los datos son importantes para las organizaciones, ya que son la base para la creación de información”. (Camisón, 2001).

En ese orden de ideas, teniendo un entorno totalmente inestable e incierto, los datos son importantes para la gestión de cualquier organización. Obviamente que hay organizaciones que requieren gestionar mayor cantidad de datos en tiempo real, dado que la naturaleza de su actividad lo exige así. Por ejemplo, las entidades bancarias y financieras; entidades públicas como la Superintendencia Nacional de Administración Tributaria (SUNAT), Seguro Social de Salud del Perú (ESSALUD), etc.; no se concibe ninguna de estas instituciones sin un sistema que permita fluir datos para sus miles de operaciones diarias.

\section{INFORMACIÓN}

La información es un conjunto de datos que dan forma a un mensaje, normalmente bajo la forma de un documento o algún tipo de comunicación audiovisual. Es el nexo que se establece entre el emisor y el receptor. La información es capaz de cambiar la forma en que el receptor percibe algo, es capaz de impactar sobre sus juicios de valor y comportamientos. En suma, una información de interés para la persona que recepciona puede modificar sus actitudes y aptitudes. A diferencia de los datos, la información tiene significado (relevancia y propósito). No sólo puede informar potencialmente al que la recibe, sino que está organizada para algún propósito. Los datos se convierten en información cuando su creador les añade significado (Arroyo, 2001).

Ahora bien, a nivel organizacional, para recepcionar o enviar información necesitamos implementar redes o sistemas de comunicación que garanticen a quien tome dicha información una acción sinérgica para la proactividad en su desempeño laboral. El tema es coadyuvar al desarrollo organizacional de modo tal que la información que circule por las redes internas sirva para ello. Los ordenadores nos pueden ayudar a añadir valor y transformar datos en información, pero es muy difícil que nos puedan ayudar a analizar el contexto de dicha información. En definitiva, que actualmente tengamos acceso a más tecnologías de la información no implica que hayamos mejorado nuestro nivel de información (Camisón, 2001).

\section{CONOCIMIENTO}

El conocimiento es una capacidad humana personal como la habilidad y la inteligencia, que se adquiere a través de la experiencia vivida. Para ello hacemos uso de nuestros sentidos, pero mucho cuidado: los materiales que nos sirven para leer, escuchar o ver no son conocimientos, son vehículos que nos sirven para internalizar la información que al aplicarlos en un hecho concreto se convertirán en conocimiento.

Para Davenport y Prusak (1999):

“... el conocimiento es una mezcla de experiencia, valores, información y 'saber hacer' que sirve como marco para la incorporación de nuevas experiencias e información, y es útil para la acción. Se origina y se aplica en la mente de los conocedores. En las organizaciones con frecuencia no sólo se encuentra dentro de documentos o almacenes de datos, sino que también está en rutinas organizativas, procesos, prácticas, y normas”.

El conocimiento es identificar, estructurar y sobre todo utilizar la información para obtener un resultado. Una persona recepciona información y le adiciona intuición y sabiduría, convirtiéndola en conocimiento.

"Las notas musicales son datos organizados de forma estructurada y coherente dentro de un contexto, con un fin. Es el conocimiento del pianista, su sabiduría, lo que hace que los datos, la información, se conviertan en verdadera melodía”. (De la Rica, 2001).

De modo que el conocimiento se deriva de la información, así como la información se deriva de los datos. Para que la información se convierta en conocimiento debe ser aplicada por las personas en su quehacer, ejecutando actividades para sí o para terceros. 
Hemos manifestado que el conocimiento es inherente a la persona, es decir, se convierte en tal cuando la persona que lo asimila como resultado de su propia experiencia, lo incorpora a su acervo personal, convencido de su significado e implicaciones. Una vez internalizado e incorporado a la estructura psicosocial de la persona, el conocimiento es inacabable. Se puede volver ha utilizar dicho conocimiento sin que se consuma, lo que no ocurre con otros tipos de bienes materiales.

El conocimiento sirve de guía para la acción de las personas, en el sentido de decidir qué hacer en cada momento, porque esa acción tiene por objetivo mejorar las consecuencias de los fenómenos percibidos por cada individuo, llegando incluso a cambiarlos de ser posible.

Ahora bien, así como las personas adquieren nuevos conocimientos a través de sus experiencias vividas, es de suponer que también las organizaciones, durante el tiempo de su existencia, adquieren y acumulan conocimientos y situación que les permiten seguir manteniéndose en el mercado. Tales conocimientos se manifiestan en el caso de las empresas en el perfeccionamiento o lanzamiento de nuevos productos o servicios, en la mejora de sus procesos, capacidad reactiva para enfrentar las contingencias, etc.

\section{GESTIÓN DEL CONOCIMIENTO}

Existen una variedad de posiciones en el mundo académico para argumentar qué se entiende por Gestión del Conocimiento. Veamos algunas de ellas:

"La gestión del conocimiento es el conjunto de procesos y sistemas que permiten que el capital intelectual de una organización aumente de forma significativa, mediante la gestión de sus capacidades de resolución de problemas de forma eficiente (en el menor tiempo posible), con el objetivo final de generar ventajas competitivas sostenibles en el tiempo". (Carrión, 2001).

"La gestión del conocimiento implica gestionar el conocimiento de la gente que directa o indirectamente tiene relación con la empresa. Dicha gestión se desarrolla sobre lo que las personas piensan y desean que se haga en la empresa para la cual trabajan, obteniendo una optimización de sus productos o servicios". (Flores, 2001).
"La gestión del conocimiento es la combinación de sinergias entre datos, información, sistemas de información y la capacidad creativa e innovadora de seres humanos". (Malhotra, 1997).

A nuestro entender la Gestión del Conocimiento es la gestión de los activos intangibles que tiene una organización para añadirle valor. Tales activos intangibles lo conforman: el capital humano, representado por el conjunto de conocimientos y capacidades de sus trabajadores; por aquellos conocimientos acumulados por la empresa en el tiempo de su existencia que se manifiesten en su know-how, patentes, marcas, etc.; y por el conjunto de relaciones que mantienen con el exterior, principalmente clientes, proveedores y otros agentes económicos, etc.

En el mundo empresarial son dos las tendencias que se vienen acentuando en el proceso de Gestión del Conocimiento:

- Tendencia de Personificación. Se sigue una estrategia de cara a cara. Se asume que el conocimiento está fuertemente ligado a la persona que lo desarrolla, y su forma de compartir será solo a través del contacto directo entre las personas. De acuerdo a esta tendencia, las tecnologías deben servir para ayudar a los trabajadores a comunicar sus conocimientos más no a almacenarlos.

- Tendencia de Codificación. Las organizaciones desarrollan vías para codificar y almacenar en bases de datos el conocimiento adquirido, de forma tal que pueda ser accesado fácilmente por cualquier miembro en el futuro. Al ser extraído el conocimiento se hace independiente de la persona que lo genera y se plasma en un soporte documental para que pueda ser reutilizado.

No siendo el espíritu de esta investigación analizar las ventajas y desventajas de tales tendencias para las organizaciones de nuestro medio, creo oportuno como introducción en la implantación del modelo de Gestión del Conocimiento, generar un sistema en la empresa que en combinación del capital humano que dispone, los conocimientos acumulados y el conjunto de relaciones que mantiene, nos permita obtener una ventaja en relación a la competencia. Si logramos embarcarnos en este proyecto habremos roto nuestras ataduras con el pasado (paradigmas tradicionales) eligiendo el camino de la gestión 
del conocimiento, que por cierto obligará a reestructurar abismalmente nuestra manera de pensar y percibir, principalmente en relación a la actuación de aquellos trabajadores que permanecen decenas de años en la empresa.

\section{PROCESO DE CREACIÓN DEL CONOCIMIENTO EN LA ORGANIZACION}

Según Nonaka y Takeuchi (1995) el conocimiento humano tiene dos variantes: Conocimiento Tácito y Conocimiento Explícito. El Conocimiento Tácito es el conocimiento inherente que tiene toda persona, producto de su experiencia, sabiduría y creatividad; en suma, conocimiento que se encuentra en el interior de cada uno de nosotros acumulado en el tiempo, el que nos proporciona la ventaja diferencial en relación a los demás. En tanto que el Conocimiento Explícito es el conocimiento que permanece en los materiales audiovisuales (libros, revistas, videos, cds, y otros) en calidad de información, que al tomar en contacto con la persona interesada se convierte en conocimiento. Este conocimiento explícito, al ser internalizado y aplicado por la persona, se convierte en conocimiento tácito. Pero como el interés de buscar nuevos conocimientos es propio de la naturaleza humana, el proceso de creación del conocimiento se convierte en un espiral que solo termina con la desaparición física de la persona (Ver Gráfico $\mathrm{N}^{\circ} 1$ ).

Gráfico № 1. Proceso de conversion del conocimiento en la organización. Nonaka y Takeuchi (1995).

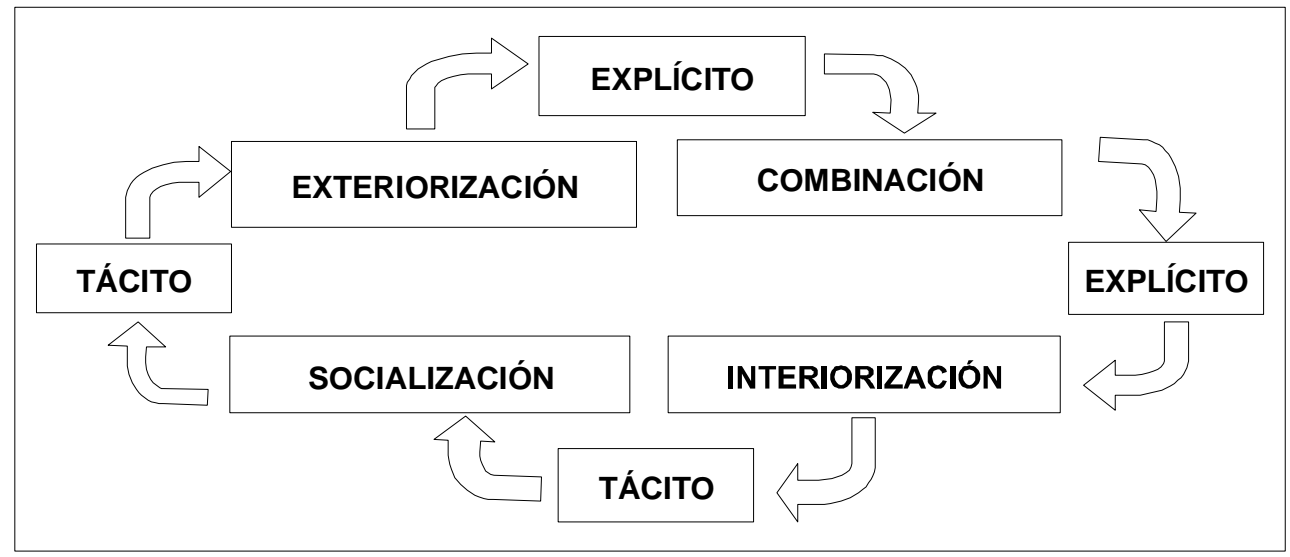

Esta combinación ontológica del conocimiento tácito con el conocimiento explícito planteado por los autores de la referencia, conocido como el modelo "Espiral del Conocimiento" conduce a la generación de cuatro campos que se convierten en fases en el proceso de creación del conocimiento.

- Socialización. Es el proceso de adquirir conocimiento tácito a partir del conocimiento tácito, mediante la interacción directa de persona a persona. Por ejemplo, el docente enseñando a sus alumnos algún modelo matemático; el albañil enseñando a su ayudante cómo asentar ladrillos; el matricero enseñando al aprendiz cómo manipular un torno para trabajar los moldes, etc. Es un conocimiento que permite compartir experiencias directas entre el poseedor y el que busca conocer y aprender.

- Exteriorización. Es el proceso de convertir los conocimientos tácitos en conocimientos explícitos. Externalizar es convertir ideas, imágenes y/o palabras a través del diálogo. Supone hacer tangible el conocimiento mediante el uso de metáforas. Por ejemplo, con este artículo mis conocimientos tácitos pretendo trasladarlos hacia mis lectores convirtiéndolos en conocimientos explícitos.

- Combinación. Es el proceso de transferencia del conocimiento explícito al conocimiento explícito. Por ejemplo, para escribir este artículo (conocimiento explícito) fue necesario consultar los trabajos de connotados autores en el tema como Drucker, Nonaka, Takeuchi, Davenport, etc., quienes a su vez transfieren conocimiento explícito.

- Interiorización. Es un proceso de incorporación de conocimiento explícito en conocimiento tácito. Por ejemplo, un viaje al exterior puede significar vivir otras experiencias, conocer otros países, diferentes culturas. Di- 
cho conocimientos se impregnan en la persona en forma de modelos mentales (conocimiento tácito) para ser utilizados en otro momento o situación.

\section{PLANTEAMIENTO DE HIPÓTESIS DE LA INVESTIGACIÓN}

Para la presente investigación, se ha creído pertinente plantear las hipótesis siguientes:

- $\quad$ Si se gerencian las Micro y Pequeñas Empresas (MYPEs) sobre la base de los conocimientos acumulados, éstas se pueden convertir en fuentes de ventaja competitiva para desarrollar las mismas en el tiempo.

- La competitividad de las MYPEs textiles ubicadas en el emporio Comercial de Gamarra está determinada, entre otros, por la capacitación permanente de sus colaboradores, uso de nuevas tecnologías y capacidad gerencial.

\section{RESULTADOS DE LA INVESTIGACIÓN}

Los siguientes son los comentarios de los resultados construidos a partir de las encuestas y entrevistas realizadas a los colaboradores y propietarios de las MYPEs de confecciones en el emporio comercial de Gamarra.

El 58.1\% de las empresas MYPEs entrevistadas perciben que no tienen nada de equipamiento o que están casi equipadas, en tanto que el $22.6 \%$ se encuentran medianamente equipadas. En este contexto, (ver Cuadro № 1) solo el $19.4 \%$ considera que los talleres se encuentran debidamente equipados.

Cuadro № 1 . Nivel de equipamiento de la MYPE.

\begin{tabular}{|ll|r|r|r|r|}
\hline & Frecuencia & (\%) & $\begin{array}{c}\text { (\%) } \\
\text { válido }\end{array}$ & $\begin{array}{c}\text { (\%) } \\
\text { acumulado }\end{array}$ \\
\hline Válidos & Nada equipado & 23 & 24,7 & 24,7 & 24,7 \\
& Casi equipado & 31 & 33,3 & 33,3 & 58,1 \\
& Median, equipado & 21 & 22,6 & 22,6 & 80,6 \\
& Muy equipado & 18 & 19,4 & 19,4 & 100,0 \\
& Total & $\mathbf{9 3}$ & $\mathbf{1 0 0 , 0}$ & $\mathbf{1 0 0 , 0}$ & \\
\hline
\end{tabular}

En lo relacionado al nivel de formación del directivo y/o propietario de la MYPE (ver Cuadro № 2), el 9\% de los empresarios tienen educación superior universitaria, en tanto que el 32\% tie- nen superior no universitaria. Como contraparte, el 59\% de los empresarios tienen entre primaria, secundaria incompleta y completa.

Cuadro № 2. Nivel de formación académica de los directivos / propietarios DE MYPES.

\begin{tabular}{|ll|r|r|r|r|}
\hline & Frecuencia & (\%) & \multicolumn{1}{|c|}{$\begin{array}{c}\text { (\%) } \\
\text { válido }\end{array}$} & $\begin{array}{c}\text { (\%) } \\
\text { acumulado }\end{array}$ \\
\hline Válidos & Secund. incomp. prim. & 21 & 22,6 & 22,6 & 22,6 \\
& Secundaria completa & 34 & 36,6 & 36,6 & 59,1 \\
& Superior no Universt. & 30 & 32,3 & 32,3 & 91,4 \\
& Superior Universt. & 8 & 8,6 & 8,6 & 100,0 \\
Total & $\mathbf{9 3}$ & $\mathbf{1 0 0 , 0}$ & $\mathbf{1 0 0 , 0}$ & \\
\hline
\end{tabular}

Los Cuadros № 3 y № 4 están referidos al proceso de motivación que se aplica en los trabajadores y el nivel de apoyo que reciben de los empresarios MYPE para sus iniciativas personales. Así en el Cuadro $\mathrm{N}^{\circ} 3$, el 60\% opina que nunca $y / o$ a veces son motivados, en contraposición con el 40\% que manifiesta ser motivado ya sea siempre o casi siempre. De la misma forma, solo el $12 \%$ dice ser apoyado en sus iniciativas personales, en tanto que el 53\% manifiesta que nunca o a veces recibe apoyo para sus iniciativas personales. 
Cuadro № 3. Motivación para conocer y aprender más en el área de trabajo.

\begin{tabular}{|ll|r|r|r|r|}
\hline & Frecuencia & (\%) & \multicolumn{1}{c|}{$\begin{array}{c}\text { (\%) } \\
\text { válido }\end{array}$} & $\begin{array}{c}\text { (\%) } \\
\text { acumulado }\end{array}$ \\
\hline Válidos & Nunca & 18 & 19,4 & 19,4 & 19,4 \\
& A veces & 37 & 39,8 & 39,8 & 59,1 \\
& Casi siempre & 27 & 29,0 & 29,0 & 88,2 \\
Siempre & 11 & 11,8 & 11,8 & 100,0 \\
& Total & $\mathbf{9 3}$ & $\mathbf{1 0 0 , 0}$ & $\mathbf{1 0 0 , 0}$ & \\
\hline
\end{tabular}

Cuadro № 4. Nivel de apoyo de la empresa a las iniciativas personales.

\begin{tabular}{|ll|r|r|r|r|}
\hline & Frecuencia & (\%) & \multicolumn{1}{c|}{$\begin{array}{c}\text { (\%) } \\
\text { válido }\end{array}$} & $\begin{array}{c}\text { (\%) } \\
\text { acumulado }\end{array}$ \\
\hline Válidos & Nunca & 27 & 29,0 & 29,0 & 29,0 \\
& A veces & 22 & 23,7 & 23,7 & 52,7 \\
& Casi siempre & 33 & 35,5 & 35,5 & 88,2 \\
& Siempre & 11 & 11,8 & 11,8 & 100,0 \\
& Total & $\mathbf{9 3}$ & $\mathbf{1 0 0 , 0}$ & $\mathbf{1 0 0 , 0}$ & \\
\hline
\end{tabular}

La disponibilidad de la información existente para solucionar problemas en la empresa se encuentra reflejada en el Cuadro № 5 . En este cuadro solo el $18 \%$ dice que la información es permanente, en tanto que el $25 \%$ manifiesta que es medianamente permanente. Sin embargo, un $57 \%$ de los encuestados ( $16.1 \%$ nunca y $40.9 \%$ a veces) manifiestan que no hay información disponible que permitan solucionar los problemas en la organización.

Cuadro $\mathbf{N}^{\circ}$ 5. Nivel de información disponible para solucionar problemas del área de trabajo.

\begin{tabular}{|ll|r|r|r|r|}
\hline & Frecuencia & (\%) & \multicolumn{1}{|c|}{$\begin{array}{c}\text { (\%) } \\
\text { válido }\end{array}$} & $\begin{array}{c}\text { (\%) } \\
\text { acumulado }\end{array}$ \\
\hline Válidos & Ninguna & 15 & 16,1 & 16,1 & 16,1 \\
& A veces & 38 & 40,9 & 40,9 & 57,0 \\
& Poco permanente & 23 & 24,7 & 24,7 & 81,7 \\
Permanente & 17 & 18,3 & 18,3 & 100,0 \\
Total & $\mathbf{9 3}$ & $\mathbf{1 0 0 , 0}$ & $\mathbf{1 0 0 , 0}$ & \\
\hline
\end{tabular}

Los siguientes son cuadros que reflejan actividades propias de la Gerencia del Conocimiento. Así, cuando se le consultó a los empresarios sobre la importancia de los conocimientos nuevos que deben adquirir los trabajadores para desarrollar bien su trabajo (ver cuadro № 6), el $65 \%$ de los encuestados consideraron que son muy importantes e importantes, en contraposición del 35\% restante que los consideraron como nada o casi importante.

Cuadro № 6. Consideración de conocimientos nuevos para desarrollar bien su trabajo.

\begin{tabular}{|ll|r|r|r|r|}
\hline & Frecuencia & (\%) & $\begin{array}{c}\text { (\%) } \\
\text { válido }\end{array}$ & $\begin{array}{c}\text { (\%) } \\
\text { acumulado }\end{array}$ \\
\hline Válidos & Nada importante & 15 & 16,1 & 16,1 & 16,1 \\
& Casi importante & 17 & 18,3 & 18,3 & 34,4 \\
& Importante & 23 & 24,7 & 24,7 & 59,1 \\
Muy importante & 38 & 40,9 & 40,9 & 100,0 \\
Total & $\mathbf{9 3}$ & $\mathbf{1 0 0 , 0}$ & $\mathbf{1 0 0 , 0}$ & \\
\hline
\end{tabular}


El Cuadro № 7 se refiere al nivel de predisposición que deben tener los trabajadores para compartir los conocimientos adquiridos. Al igual que en el cuadro anterior, el 66\% manifiesta que están predispuestos ya sea mediana o permanentemente; en tanto que el diferencial dice estar nada o casi permanentemente predispuestos a compartir sus conocimientos.

Cuadro № 7. Nivel de predisposición para compartir los conocimientos adquiridos.

\begin{tabular}{|ll|r|r|r|r|}
\hline & Frecuencia & (\%) & $\begin{array}{c}\text { (\%) } \\
\text { válido }\end{array}$ & $\begin{array}{c}\text { (\%) } \\
\text { acumulado }\end{array}$ \\
\hline Válidos & Nada permanente & 12 & 12,9 & 12,9 & 12,9 \\
& Casi permanente & 20 & 21,5 & 21,5 & 34,4 \\
& Mediante permanente & 29 & 31,2 & 31,2 & 65,6 \\
Permanente & 32 & 34,4 & 34,4 & 100,0 \\
Total & $\mathbf{9 3}$ & $\mathbf{1 0 0 , 0}$ & $\mathbf{1 0 0 , 0}$ & \\
\hline
\end{tabular}

El proceso de valoración del uso de las tecnologías modernas también fue consultado a los encuestados. Así el $82 \%$ manifestó que el uso de tecnologías modernas es muy importante y/o importante para almacenar información, a diferencia del $18 \%$ que dijeron lo contrario (ver
Cuadro № 8). Estos son concordantes con los resultados del Gráfico № 2, referido a la frecuencia de capacitación recibida para innovar los diseños de las prendas. En esto, solo el $42 \%$ manifiesta que recibió capacitación, en tanto que el $58 \%$ dice lo contrario.

Cuadro $\mathrm{N}^{\circ}$ 8. Valoración del uso de tecnologías modernas para almacenar información.

\begin{tabular}{|ll|r|r|r|r|}
\hline & Frecuencia & (\%) & \multicolumn{1}{|c|}{$\begin{array}{c}\text { (\%) } \\
\text { válido }\end{array}$} & $\begin{array}{c}\text { (\%) } \\
\text { acumulado }\end{array}$ \\
\hline Válidos & Nada importante & 5 & 5,4 & 5,4 & 5,4 \\
& Casi importante & 12 & 12,9 & 12,9 & 18,3 \\
& Importante & 33 & 35,5 & 35,5 & 53.8 \\
Muy importante & 43 & 46,2 & 46,2 & 100,0 \\
Total & $\mathbf{9 3}$ & $\mathbf{1 0 0 , 0}$ & $\mathbf{1 0 0 , 0}$ & \\
\hline
\end{tabular}

Gráfico $\mathbf{N}^{\circ}$ 2. Frecuencia de capacitacion recibida para innovar productos.

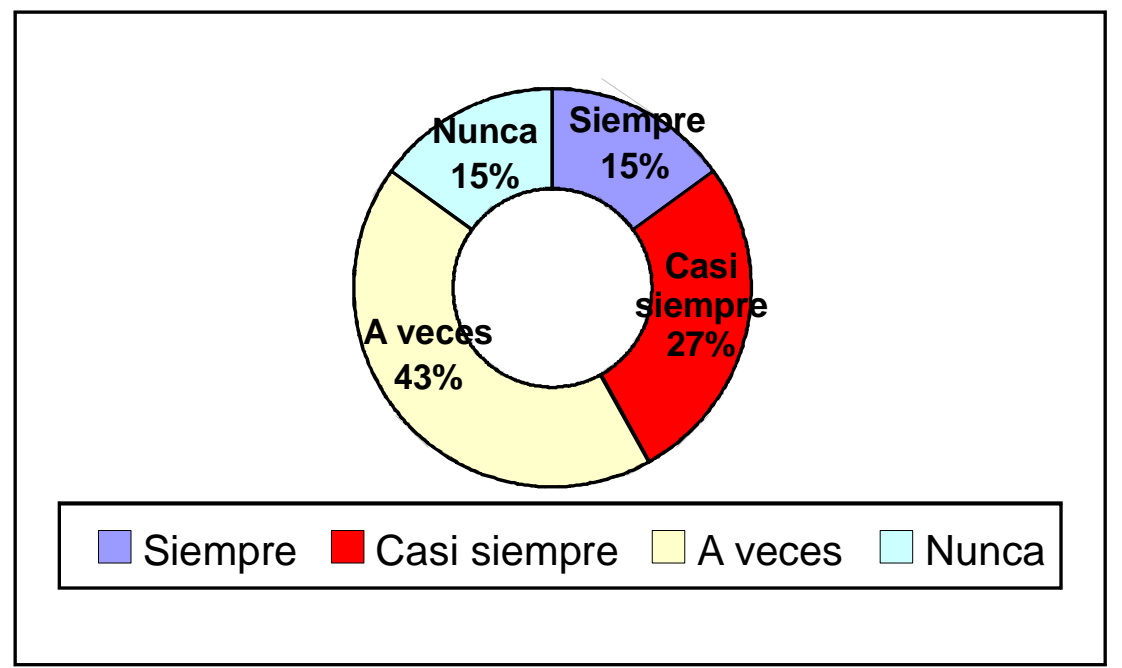

Finalmente, en relación al nivel de adaptación a los cambios, el Gráfico № 3 muestra que el $52 \%$ de los encuestados está dispuesto a adap- tarse con facilidad, en tanto que el $48 \%$ restante manifiesta que lo harían con poca facilidad. 
Gráfico $\mathbf{N}^{\circ}$ 3. Nivel de adaptacion a los cambios en la empresa.

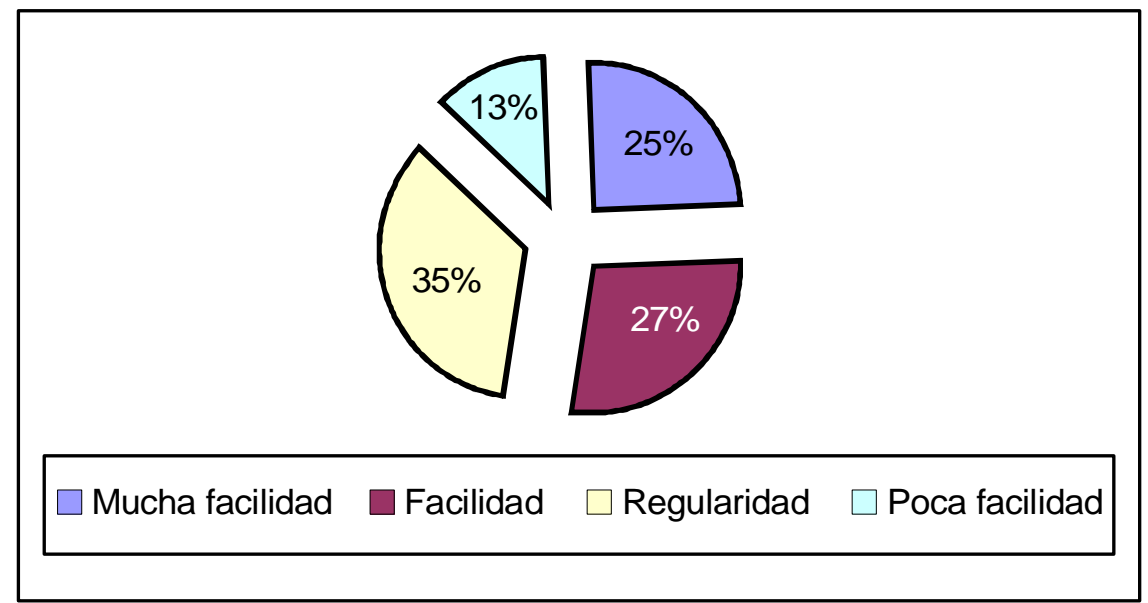

\section{ANÁLISIS DE LA INVESTIGACIÓN}

Los resultados del trabajo de campo realizado en los colaboradores y directivos de MYPE de confecciones del emporio comercial de Gamarra, a través de las encuestas y entrevistas, arrojan, entre otros resultados que todavía no están preparados para gestionar sus empresas sobre la base de los conocimientos acumulados en el tiempo. Así, a pesar de que la mayoría de los colaboradores están predispuestos a compartir información, sin embargo, el clima laboral así como el estilo gerencial que aplican los propietarios por ahora no facilitan ese proceso. De la misma forma, tampoco existe el nivel de formación académica y conciencia en los propietarios de los talleres para sistematizar a través de alguna tecnología informática moderna (por ejemplo, base de datos), los conocimientos que con el correr del tiempo vienen acumulando.

Sin embargo, cuando se consultó acerca de la importancia que tienen los nuevos conocimientos, la democratización de los mismos entre compañeros de trabajo, así como por el uso intensivo de tecnologías modernas en el diseño y fabricación de nuevos modelos y prendas, las valoraciones a tales estrategias fueron muy altas, lo cual es coherente si tenemos en cuenta que las confecciones, por el efecto de la moda, son un sector altamente competitivo.

\section{CONCLUSIONES}

Después de haber realizado esta investigación, en los empresarios de la Micro y Pequeña Empresa del emporio comercial de Gamarra, a modo de conclusión manifestamos lo siguiente:
1. La Gerencia del Conocimiento es un modelo de gestión que permite desarrollar a las organizaciones empresariales sean éstas micro, pequeñas, medianas o grandes empresas. A diferencia de los últimos, creemos que en las micro y pequeñas empresas, dadas sus limitaciones financieras para la implementación de este modelo deben seguir la tendencia a la personificación; vale decir, una gestión caracterizada por el contacto permanente con sus colaboradores donde la tecnología ayude a comunicar sus conocimientos más no a almacenarlos.

2. Todavía no están dadas las condiciones clima laboral en los talleres y formación académica de los propietarios- para gerenciar las MYPEs sobre la base de los conocimientos acumulados a través de sus colaboradores. Esto se refleja en el celo que todavía subsiste entre los trabajadores, dificultando compartir conocimientos, habilidades y destrezas, aunque están convencidos que para desarrollar las organizaciones necesariamente deben interactuar e intercambiar conocimientos.

3. De la misma forma, a partir de los resultados de la investigación, se concluye que la adquisición de nuevos conocimientos para desarrollar su trabajo tiene una alta valoración entre los trabajadores y propietarios de las MYPEs. Creemos que este aspecto se debe capitalizar para internalizar los valores de interacción humana y trabajo en equipo.

4. En suma, ante estas nuevas "reglas del juego", la empresa que aproveche las capaci- 
dades intelectuales de la organización, que desarrolle su capacidad de aprendizaje, que potencie la innovación constante y la creación de nuevos conocimientos y que desarrolle sistemas y tecnología necesarios para ello, estará en situación de afrontar los retos futuros. Capturar y gestionar estas capacidades y conocimientos y rentabilizarlos, en definitiva, convertir el capital intelectual en capital financiero, es el nuevo paradigma empresarial de estos tiempos.

\section{BIBLIOGRAFÍA}

Arroyo, A. (2001). ¿Conoce el Significado e Implicación de la Gestión del Conocimiento?. En: http://www.revista.robotikev.com/gc/n.

Bueno, E. (1998). El Capital Intangible como Clave Estratégica en la Competencia Actual. En: http://www.gestiondelconocimiento.com/ index.php3.

Camisón, C; Palacios, D; Devece, C. (2001). Gerencia del Conocimiento. En: http:// www.gestiondelconocimiento.com/ artículos.htm.

De la Rica, E. (2001). Gestión del Conocimiento: Caminando hacia las organizaciones inteligentes. En: http://www.nuevaempresa.cu/ 03_21.thm.

Davenport, T. y Prusak, L. (1999). El Conocimiento activo: Cómo las Organizaciones manejan lo que ellos saben. En: http://www.gestiondel conocimiento.com/bibliografía_ conceptos.htm

Drucker, P. (1995). Los desafíos de la Administración en el Siglo XXI. Argentina, Edit. Sudamericana.

Drucker, P. (1999). Los desafíos de la Gerencia para el Siglo XXI. Barcelona, Editorial Norma.

Malhotra, Y. (1997). La dirección de conocimiento en la organización inteligente. Procedimientos de la Asociación para los Sistemas de Información. Tercera Conferencia Americana en los Sistemas de Información. En: http:/ /www.brint.com/Km/Km.htm.

Nonaka, I; Takeouchi, H. (1995). La Empresa creadora del Conocimiento: Cómo las Compañías japonesas crean la dinámica para la Innovación. Edit. Universidad de Oxford.

Moreno, Luis. (1995). Gamarra, una mirada hacia el futuro. Lima.

Gonzales, Julia. (2001). Redes de la informalidad en Gamarra. Lima, Edit. Univ. Ricardo Palma.

Sánchez, Humberto. (2004). Gamarra: Hacia la conquista del mercado exterior. Lima, Edit. Capymex.

Puzueta de Paredes, F. (2000). Los primeros pasos hacia la Gestión del Conocimiento. En: http:/ /www.gestiondelconocimiento.com/ casos_area_profesional. 